\title{
A STRATEGY FOR AUTOGENERATION OF SPACE SHUTTLE GROUND PROCESSING SIMULATION MODELS FOR PROJECT MAKESPAN ESTIMATION
}

\author{
Michael G. Madden \\ Roberta Wyrick \\ Dale E. O’Neill \\ United Space Alliance, LLC \\ Florida Program Office \\ 8550 Astronaut Blvd \\ Cape Canaveral, FL 32920, U.S.A.
}

\begin{abstract}
Space Shuttle Processing is a complicated and highly variable project. The planning and scheduling problem, categorized as a Resource Constrained - Stochastic Project Scheduling Problem (RC-SPSP), has a great deal of variability in the Orbiter Processing Facility (OPF) process flow from one flight to the next. Simulation Modeling is a useful tool in estimation of the makespan of the overall process. However, simulation requires a model to be developed, which itself is a labor and time consuming effort. With such a dynamic process, often the model would potentially be out of synchronization with the actual process, limiting the applicability of the simulation answers in solving the actual estimation problem. Integration of TEAMS model enabling software with our existing schedule program software is the basis of our solution. This paper explains the approach used to develop an auto-generated simulation model from planning and schedule efforts and available data.
\end{abstract}

\section{INTRODUCTION}

Space Shuttle ground processing is a complicated series of tasks. The Space Transportation System (STS) or Space Shuttle is made up of three major elements: the Orbiter or spacecraft, the External Tank (ET), and the Solid Rocket Boosters (SRB). Processing of the spacecraft is performed sequentially in three major facilities during preparation for flight: the Orbiter Processing Facility (OPF), the Vertical Assembly Building (VAB), and the launch pad (PAD). The majority of the spacecraft ground processing occurs in the OPF in a horizontal orientation like an airplane, as shown in Figure 1. The processing tasks performed in the OPF include: removing the previous mission's payload hardware, inspection of the tile and vehicle for the next flight, configuration of the vehicle to support the next mission, modification of the vehicle with any upgrades or new hardware, system level testing of all functions for the next flight, integrated testing of multiple systems per published requirements, and resolution of all problems and damage identified before the next mission. Many of the tasks are hazardous involving toxic or explosive materials, confined spaces, suspended loads, working at heights, and radiation exposure. This process takes about three months to complete for standard processing and is the portion of the overall process with the most variability. A discrete event process simulation model is highly desired for timeline analysis and estimation of flow durations. However, it would be difficult to initially create the simulation model manually and to maintain it during the execution of the process.

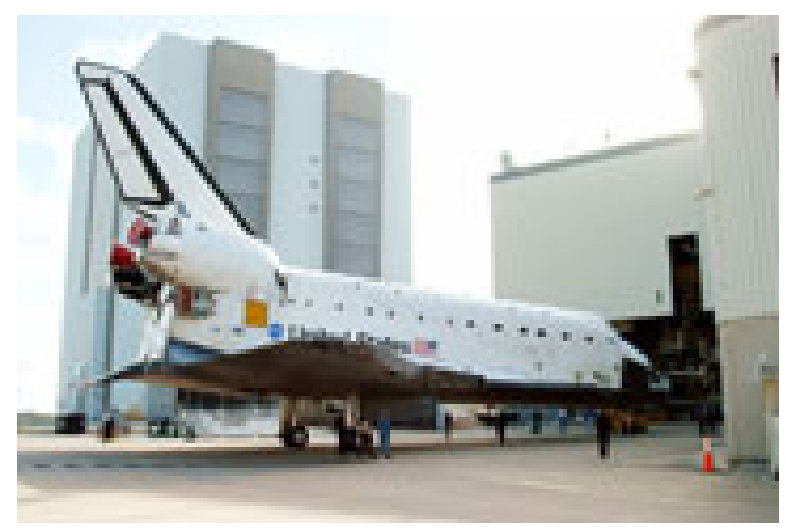

Figure 1: Space Shuttle Atlantis Rolling into OPF Bay 1 for Flow Processing after a Storage Period.

The OPF flow process is conceptualized similar to a new, small construction project in duration and format with tasks requiring different skills and actions at varying times during the flow. Some tasks must be performed in a precedence network fashion while others can be performed in parallel only being limited by available resources and area access. Still other tasks are time-dependent and must 
be performed either very early or very late in the OPF process. There is another group of tasks which are configuration-dependent, meaning that the spacecraft must be in a certain state for the performance of that task. This situation requires the scheduling of additional operations in the process to establish the needed spacecraft configuration. The scheduling tool currently used by the Space Shuttle Program (SSP) contains this level of task effect and requirement details for each operation. More details on the scheduling software and associated algorithms can be found in (Zweben, Davis, Daun, \& Deale, 1993).

There is a great deal of variability in the OPF process flow from one mission to the next. Initially, all flows are planned with a generic outline and as the time gets closer to the actual flow starting point (landing of the previous mission), more and more specific content of the processing flow becomes known, understood and planned into the schedule. Even after the flow has begun, there is still a great deal of variability since many of the tasks are inspections and system tests which have the potential to locate damage or problems. These issues must then be repaired or resolved before the next launch of the spacecraft. Approximately $30-60 \%$ of the work performed is unknown at the beginning of the flow. This phenomenon is not unusual for depot maintenance requirements and checkout of aircraft. The literature contains references to similar levels of variability in aircraft overhaul and repair. (Boydstun, Graul, Benjamin, \& Painter, 2002)

The great variability of the OPF process makes the planning and estimation of the actual completion date a challenge. Although the schedule and manifest have a fixed amount of time identified (Rollout Date and Launch Date are known) for each OPF flow once started, it is difficult for managers to know when added "unknown work" volume has "broken the back" of the initial schedule commitments and agreements. Modeling and Simulation would be a very useful tool in estimation of the completion of the process, but modeling and simulation requires a model to be developed and this itself is a labor and time consuming effort. With such a dynamic process, often the model would be out of sync with the actual process, limiting the applicability of the simulation answers in solving the actual estimation problem. The question posed by USA and NASA management is this: "Can an efficient method be developed to utilize the existing efforts of scheduling for an OPF Processing Flow makespan analysis?"

The remainder of this paper is organized with a problem statement in section 2, a review of relevant literature in section 3 , a proposed solution in section 4 , a description of methodology used and verification and validation techniques in section 5 , our conclusions in section 6 and identification of future research areas in section 7 .

\section{PROBLEM STATEMENT}

The major objective of this project is to produce an accurate basis of estimation of OPF flow makespan. Discrete event simulation is the chosen method of analysis and automatic generation of the model is the desired implementation technique to take advantage of the planning and scheduling effort that already exists. Careful utilization of databases and expert knowledge within the schedule product dataset will produce the simulation model without a unique modeling effort for all iterations of the new schedule.

The planning effort associated with shuttle processing produces data that is very similar to simulation model development information and structure. Planners and schedulers daily evaluate newly added tasks for each OPF flow, shown in Figure 2. The planning tools have a large amount of process information contained in them and, if properly connected together with historical data of similar processes and tasks, could represent a basis for automatic generation of a simulation model. Originally designed for automated scheduling deconfliction, the Ground Processing Scheduling System (GPSS) (Zweben et al., 1993) planning tool used by the SSP to schedule daily operations is no longer automatically performing this function, but the data is still maintained in the system. In an effort to develop this capability, we started working with the model enabling technology of the Toolkit for Enabling Automated Modeling and Simulation (TEAMS) effort between NASA and KBSI. (Benjamin, Graul, \& Erraguntla, 2002)

\section{USA Shuttle Processing Planning Products}

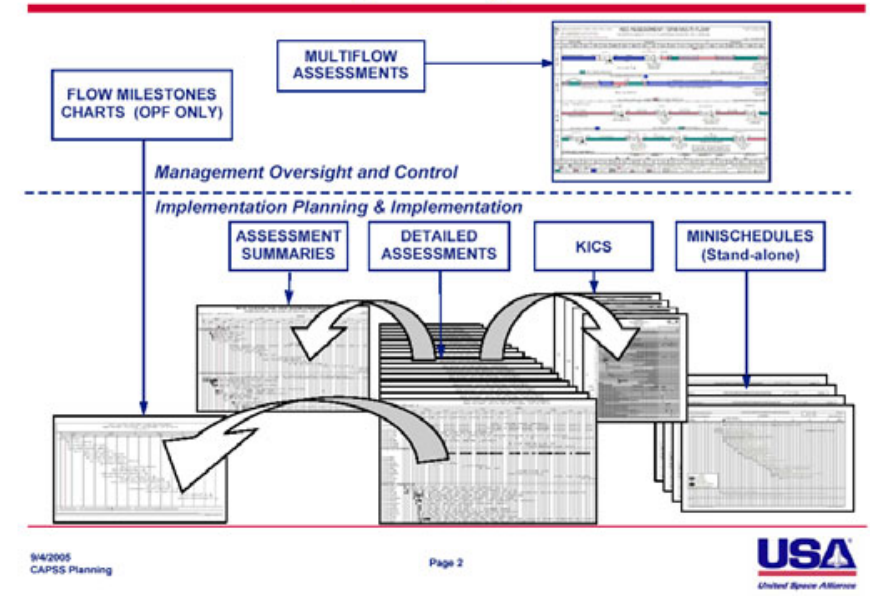

Figure 2: Various Levels of Planning and Scheduling of Activities for OPF Flow Processing

Planners and schedulers daily evaluate newly added tasks for each OPF flow and their efforts input the details of these tasks into an electronic format that could be accessed 
by a simulation model automatic creation program. This concept would leverage the labor efforts of the existing planners and schedulers into model development without adding headcount, while at the same time keeping the model up-to-date with all the latest variability in the process known at the time.

Traditionally, the overall estimation of the OPF process has been limited to the expertise and knowledge of Senior Planners and Flow Managers. Their techniques utilize extensive years of experience and corporate knowledge based estimates that are often very accurate in their predictions. However, as time goes by, employees leave NASA and USA, taking their knowledge and experience with them. In addition, NASA is requesting more formal and precise basis of estimates for the flow duration. This evolution instigated the need for a modeling and simulation solution. When initiating a flow, the variability of the process and the amount of unknowns do not lend themselves to closed form solutions for determining total duration (makespan) of the project. Unlike a new construction project, where most of the tasks are known but the durations are subject to variability, the shuttle process has task content variability in addition to duration variability. Questions like "How many problem reports will be initiated and how extensive will the repairs be before the flow is complete?" are very hard to precisely predict and model.

\section{LITERATURE REVIEW}

In (Brucker, Drexl, Möhring, Neumann, \& Pesch, 1999), a scheme for the classification of resource-constrained project scheduling problems (RC-PSP) is identified. Using the notation of Brucker, et al. A description of the OPF Processing flow would be as follows:

$$
\text { PS - } m, \sigma, \rho \mid p_{j}=\text { sto, d, prec } \mid C_{\max }
$$

This indicates a Project Scheduling Problem - with $\mathrm{m}$ - renewable resources with $\sigma$ units available, and each activity requires at most $\rho$ resource units. The approximate number of tasks or activities at the start of the project is 500 , increasing to approximately 2000 by project completion. The resources (mostly technicians) are renewed each day/shift and thus are considered renewable resources. In addition, there are limited numbers of certain ground support equipment (GSE) items which are also modeled as resources for this project.

The activity characteristics indicated in the second term are as follows: processing times are stochastic $-\left(\mathrm{p}_{\mathrm{j}}=\right.$ sto), there is a scheduled due date $-\mathrm{d}$, and many tasks are subjected to a precedence network - prec. The third term represents the objective function, and the author has chosen it as minimum makespan $-\mathrm{C}_{\max }$. This choice is the initial selection of this modeling project as there is a desire to develop a sound basis of estimate for the process makespan. After an automatic modeling tool has been developed and the model can be verified and validated, other objective functions will be considered, such as minimization of Early-Tardy penalties and resource leveling. However, the initial goal is to simply understand and estimate the OPF flow process makespan.

The notation does not appear to provide a way of characterizing the configuration state effects and requirements. A Petri-Net solution may be better able to handle the added dimension of our specific scheduling problem and the state requirements. Initially, we are attempting to treat the states as psuedo-resources made available by performance of other activity.

Further examination of the (Brucker et al., 1999) paper in section 8 concerning Stochastic Activity Durations indicates the complexity and mathematical errors common to this type of RC-PSP. "There is a systematic under estimation of the project completion timeline...if one compares 'deterministic makespan' obtained from expected processing times with the expected makespan even in the absence of resource and state constraints."

When the concept of resource constraints is added to the problem, research indicates that it is best handled by a series of policies and strategies, rather than a closed form optimization technique. A complete characterization of the policies and subclasses has been given by (Möhring \& Stork, 2000). This research indicates that policy strategies are good solutions to this type of RC-PSP problem.

With respect to the subject of Critical Chain Scheduling (Herroelen \& Leus, 2001) state: "Nevertheless, for single projects, the unconditional focus on a 'critical chain' seems useless to us: it obscures extra scheduling options, and enforces a rigid focus on what was critical at the start of the project but may no longer be crucial after a certain lapse of time. Makespan estimation is approximate anyway because of the merge bias and the simplification of the resolution of resource conflicts. Practical makespan estimations can be obtained based on scenario analysis, and/or the incorporation of an aggregate protection measure under the form of a project buffer alone. The experience of the scheduler will have the final word. The durations are often based on the behaviour of human resources; so one should not rely on overly sophisticated statistical techniques for modeling them (for one, because their variability can be influenced by management)."

From (Pritsker, Sigal, \& Hammesfahr, 1989): Pritsker et al. pointed out that after a sufficient number of simulation runs, a ranking of the activities by high value of average slack (TF) time becomes a possible method for ordering the activities that can be delayed. A more appropriate ranking is based on the ratio of the average slack time to the standard deviation of activity duration.

$$
\text { Rank }=\mathrm{TF} / \text { Std. Deviation of Task Variability }
$$


A higher ratio value indicates that there is less likelihood that the average slack time will be exceeded due to the value of the basic variability inherent in the performance of the activity. A low ratio value indicates there is minimum latitude in the start time for the activity. The above analysis (2) demands some in-depth knowledge of statistics and is not easy for the common practitioner to use.

In (Pritsker et al., 1989) the final conclusion stated that "there is a large positive correlation between the ranking of critical activities based on the ratio of average slack (TF) to activity duration standard deviation and the criticality index." Criticality index (CI) for an activity in a percentage term is defined as the number of simulation runs in which the activity is critical, divided by the total number of simulation runs (3):

$$
C I=\frac{n \text { Critical Runs }}{\text { Total Runs }}
$$

The ratio and formula (3) is similar to an idea our team had for improving the space shuttle scheduling process. Our idea, or policy theory, is that for parallel tasks, where all other discriminators are equal, basing the order of performance on the tasks with the highest variability first will have an effect of shortening the schedule. This is a policy theory, and once a modeling system has been developed and validated, a secondary objective for our team will be to use this type of problem (OPF Process Flow) and the simulation model to prove the stated policy theory. This concept in Pritsker goes beyond our concept to provide a method to re-rank items based on the total slack/variability potentially allowing tasks to be reorganized more efficiently within the precedence network. This may also be related to the AND/OR precedence concepts of several of the German based papers. In another construction and project management paper (Akpan, 2000) it is suggested that a random method of task selection produces a near optimal result. All of these will become options for our evaluation with the new simulation models afforded us by this project.

From (AbouRizk \& Wales, 1997): "In order to be accepted in the construction environment, simulation has to be presented in a very simple and graphical context. Contact with construction professionals indicates that formats which appear to be too theoretical or analytical tend not to be accepted or utilized. Therefore, ideal simulation systems should be pictorial or schematic emphasizing graphical input and graphical output. The early systems designed to study construction operations utilized simple bar charting concepts."

Just as with the construction business, the experience of our team also indicates that detailed, theoretical solutions to the SSP OPF scheduling project estimation may not be well received by management. A simplified simulation solution that can mirror the existing scheduling process by reflecting the actual planning product already collaborated, would improve the likelihood of acceptance from space program management. Our auto-generated model from the daily schedule should be a good basis for this management acceptance effort.

\section{PROPOSED SOLUTIONS}

The discrete event simulation technique will be the primary method used to solve this problem. The solution will utilize existing information from the scheduling/planning tool combined with a data repository to be built up with details from as-run OPF flow processing data, shown in Figure 3. For many years, OPF Flow process as-run information was accessed by way of a printed graphical format, a book with the schedules and the actual durations printed for each STS Flow, and retained on a shelf in the planning area. The planners referenced these printed copies of schedules anecdotally whenever a similar series of problems were identified in a current flow. The effort would be to match a particular new series of tasks to one that had previously occurred and use the timelines from that as a basis for estimating the future schedule. This history matching effort was hit or miss at best. The reliance was on the planner to know in which STS book to find a similar pattern of scheduled tasks and would then result in one or two data points from the past being loosely applied to the future schedule.

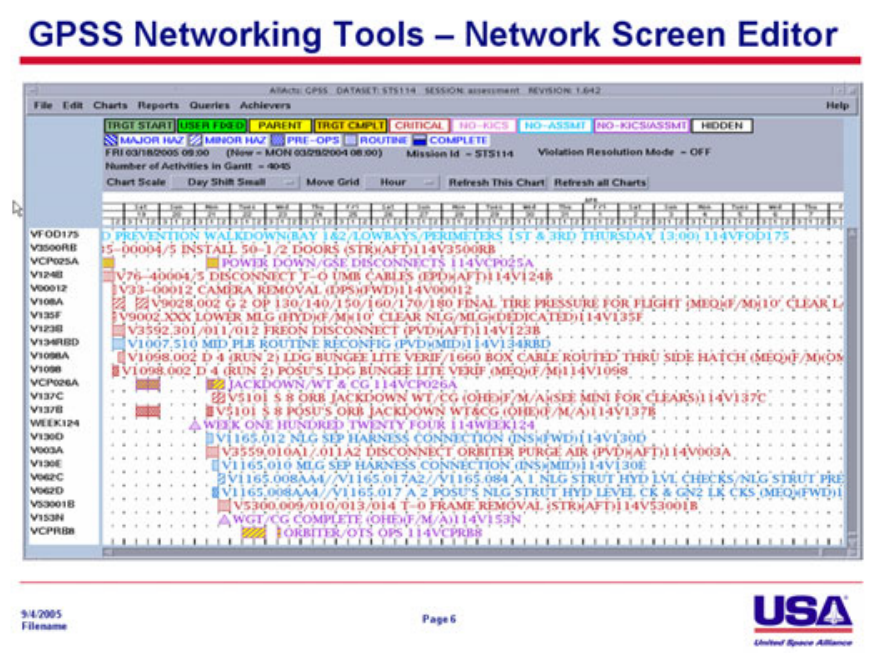

Figure 3: Screen Shot of GPSS Scheduling Software during the Daily Process of Revising the Schedule.

The breakthrough to modernize this effort came when we realized that the data to build the graphical schedules was maintained electronically and could be collected and stored together from each flow into one common analysis database. This database now contains all processing flow 
scheduled tasks from about 1990 to present date. A total of more then 160,000 task line items have been collected and stored in a database for the schedule analysis. Never before was data from different flows merged into one analyst accessible database. The details of each flow were all stored separately in their own files for the historical record. The available schedule information comes from two different computer systems. The greatest amount of details are stored in the GPSS system, but those files were only available for the last 20 flows. The other system of recording flow data is the Computer-Aided Planning and Scheduling System (CAPSS), which had less details, but was available back to STS-35 (1990 timeframe).

The data contained as-run durations of all tasks; meaning the time periods that these tasks were being worked by technicians on the daily schedule. This as-run data from history was checked and recorded daily in the schedule system by the planners and schedulers as the flows were occurring. The data contains the name of each task. If a problem was being resolved in conjunction with a pre-planned procedure; there is also a loose record of which problems were related to which planned tasks. There are also pieces of information in the database as to the start date and the end date of the task. This information can be used to determine an elapsed time period for a task. A work schedule calendar indicating the date on which each job was performed, as well as a listing of resources needed per task are included in the data. This information will be collectively analyzed to produce distribution of duration and delay for each task scheduled in OPF Flows on all space shuttles since the 1990 timeframe. The difference between the scheduled duration and the elapsed time represents delays to tasks. There is not a clear definition or reason for these delays, but for similar tasks over time, a distribution of delays for specific tasks will be developed. The assumption is that the pattern of delays in the past represent the pattern of delays in the future, without knowing line by line what the reasons were for each delay.

Once data had become a requirement for this modeling effort, other related databases were searched for additional schedule/process information. These databases shown in Figure 4, included the Problem Recording and Corrective Action (PRACA) system, which records all problems identified for each system and component during the flow. The key piece of schedule information in the PRACA database was the "detected during" field. This field, when properly completed and filled in, establishes a precedence network for scheduled tasks that identify hardware problems. A detailed analysis of this information, combined with the known schedule data, will be used to establish the probability of detecting a problem from a scheduled planned task. Several other databases which contain records of modifications and special tests performed on each flow can also be worked into the historical analysis process to better understand some of the "non-routine" processes added to each flow over time. The different types of unknown or unplanned work for aircraft are discussed very effectively in (Boydstun et al., 2002). The situation is very similar for spacecraft.

\section{Database Integration Effort}
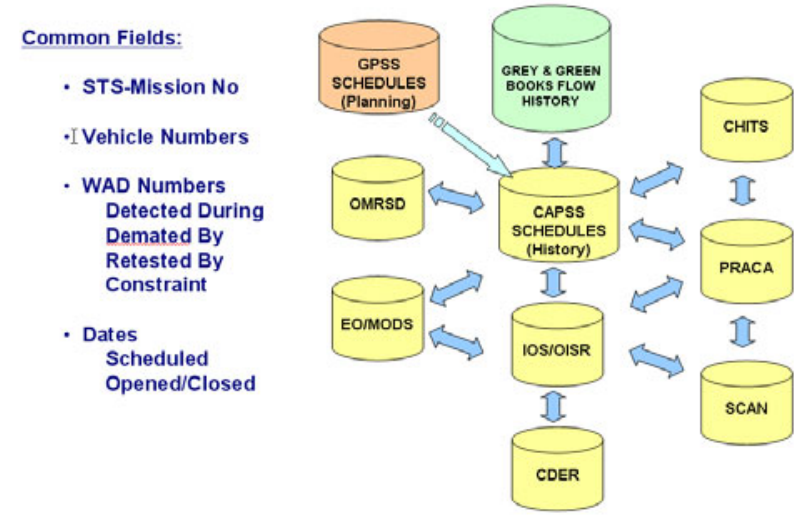

Figure 4: Multiple Database Integration Efforts Required for Determination of Modeling Details and Input Distributions

The data limitation encountered while working with different databases was the lack of consistency in some of the fields. The different systems had freeform fields for Work Authorization Document (WAD) numbers. When attempting to model and simulate processing flows, consistent usage of WAD numbers from flow to flow would be very beneficial for the analysis of the distributions. Also, the connection from one database to another relies on the same WAD number in both databases. Initially, this was not possible due to the recording of different formats for the same WAD number in the different databases. A cleanup effort was required for each data source to standardize the WAD numbering system. A field was created to input the clean version of the WAD number for database-to-database connectivity. The effort is an on-going process and is creating more and more detailed pictures of our history.

The TEAMS+ concept was developed with the help of the researchers from Knowledge Based Systems, Inc. (KBSI). KBSI was already developing technology to utilize and enable database information to support modeling for NASA. NASA requested USA to support the TEAMS effort and provide them with a sample process and data for development of model enabling technology. Through collaboration, the plan of using the schedule program data, as well as the historical data was formulated, and contracts are on-going at this time.

The initial models developed will answer questions about the OPF Flow process as to expected durations of the flow, resource utilizations, over-allocations and other general information. The plan to develop a more optimal 
schedule is not the initial goal of this simulation modeling effort, yet once developed, the model will lend itself to the evaluation of process improvements and optimization techniques in a way that could not be done by actual process changes. The model allows for a controlled environment for the experimentation of process changes which does not exist in the real world. Furthermore, due to the extended length of each flow and the low flight rate of the shuttle, a good sample of a process improvement would take several years.

NASA was already using ARENA software for higher-level process models. KBSI had already established some interfaces with ARENA models for other efforts. The KBSI tool utilizes a WorkSim tool for the modeling, but it was determined that, in addition to the KBSI tool, development of ARENA models would allow USA and NASA to customize and share simulations. This feature/benefit was of great importance to the project. USA, in an effort to increase collaboration and partnering with NASA on schedule estimates, wanted the new tool to be fully compatible with NASA's existing modeling capabilities. To this end, the development of a new tool that both sides could understand, digest and work directly with, would go a long way in the establishment of a partnering solution.

\section{METHODOLOGY}

The development of typical model constructs, known as modules, shown in Figure 5, are required to handle the conversion from schedule data into simulation model format.

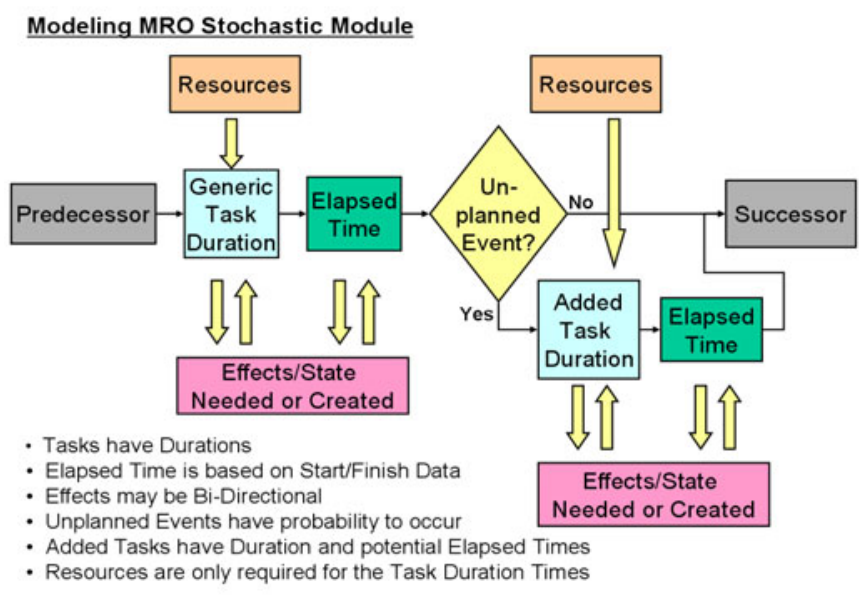

Figure 5: Basic Task Module and Simulation Construct used for Modeling of the OPF Flow Process

If we consider that each task follows a certain model structure pattern, then the data that feeds the different parts of the pattern can be assigned and extracted in the conversion of the schedule data into a simulation model package.
The data available to use for modeling includes task durations and elapsed time. The elapsed time represents the portions of the OPF process that are not explicitly available in the data. There is a significant portion of variability that is not documented directly. Delays due to design center resolutions, lack of system engineering availability, and problem resolution are some of the areas that the difference between the recorded duration and the total elapsed time in the task data are explained.

When modeling this process, concerns arise that adding delays for resource availability and these explicit elapsed time delays will have the model double booking the reality of the delays. To counteract this potential and to be able to dial the model into the accurate representation of our process, a gain factor might be considered for the elapsed time durations. This gain factor would be a variable set for the entire model that when changed was multiplied by the added elapsed times to the tasks. This might be a percentage ( $75 \%$ for example), eliminating $25 \%$ across the board for a given flow, reducing the entire makespan to more accurately represent the data.

\subsection{Notation and Distribution Calculations}

Durations representing the time that resources are captured by the activity, while 'dwell time' represents delays, which only constrain the precedence network and the configuration states.

- $\quad \mathrm{T}_{\mathrm{p}}=$ Planned Duration of Activity

Hours - Deterministic - Snapshot taken at Baseline - Roll-in of Orbiter

- $\mathrm{T}_{\mathrm{a}}=$ Actual Duration of Activity Hours - Recorded in As-Run Data for each Flow Stochastic and Discrete, meaning accuracy was recorded in Hours of 2-4 Hour blocks by shift for all flows from STS-35 to present.

- $\quad \mathrm{ET}_{\mathrm{p}}=$ Elapsed Time Planned Total Time from Planned Start to Planned Finish including Holds and Delays

- $\quad \mathrm{ET}_{\mathrm{a}}=$ Elapsed Time Actual

Total Time from Actual Start to Actual Finish including Actual Holds, Planned Holds, and Planned Delays and Actual Delays

- $\quad \mathrm{DT}_{\mathrm{p}}=$ Dwell Time Planned $\mathrm{DT}_{\mathrm{p}}=\mathrm{ET}_{\mathrm{p}}-\mathrm{T}_{\mathrm{p}}$

A Stochastic Value minus a Deterministic Value

- $\quad \mathrm{DT}_{\mathrm{a}}=$ Dwell Time Actual

$$
\mathrm{DT}_{\mathrm{a}}=\mathrm{ET}_{\mathrm{a}}-\mathrm{T}_{\mathrm{a}}
$$

Stochastic Historical Data

$$
\begin{array}{r}
\Delta \mathrm{DT}=\text { Delta Dwell Time } \\
\Delta \mathrm{DT}=\mathrm{DT}_{\mathrm{a}}-\mathrm{DT}_{\mathrm{p}}
\end{array}
$$

We intend to develop stochastic input distributions for each activity in our network. The historical data will allow 
us to determine each of the activities Ta, ETa, DTa, DTp, $\Delta \mathrm{DT}$. To determine these values for each task or activity is a challenge, because our data has occurred over a period of 20 years of processing the spacecraft. The activities have evolved over time and have different levels of checkout detail across various flows. Our data does not contain enough information to clearly know what specific sequences were performed during each flow. We know the titles of the documents and the systems that were evaluated, but whether 10,15 or 20 different sequences were performed on a given flight is not available in the dataset. Our assumption initially will be that whatever pattern of testing was conducted in the past will be the patterns of testing done in the future. If the data shows a trend with time or a bimodal type pattern then we will investigate into that specific activity more closely. Just as indicated in (Boydstun et al., 2002), hidden patterns of actual distributions may be present in our overall aggregated distributions depending on the types of checkout performed rather than the type of problems being resolved. In the long run, after developing our initial model, if we require more accurate estimations, this may be an area that we can refine with more detailed research into our history and recorded documentation.

\subsection{Data Analysis Techniques}

All 160,000 task line items are contained in a MS Access database. This number exceeds the capacity to work within one spreadsheet in MS Excel, which has a $65 \mathrm{~K}$ line limit. Several options are available to us: usage of an OLAP Cube from the data in MS Access feeding into a pivot table in MS Excel, or usage of a summation query in MS Access grouping the task pieces into a larger aggregate by flow and activity. The output from this summation query can then be exported into MS Excel for further analysis.

Once we have a manageable dataset in MS Excel, the use of the Pivot Table feature will be one of our analysis techniques. We will then have the ability to structure a table with Mission Flows going across the horizontal axis and Activities going down the vertical axis. A Visual Basic Application (VBA) macro was developed to produce an empirical distribution for each activity counting the frequency of each recorded time and produces an empirical distribution for the data similar to cdf. Initially there will be no attempt at curve fitting on this first analysis, simply a cdf of the real data points, which are recorded discretely in our dataset. The next more detailed analysis will use ExpertFit software. This software has some special techniques that perform several 'goodness of fit' type tests on the data sets and makes several recommendations of theoretical distributions which can represent the distributions in our recorded data.

Additional assumptions must be made for the transition from an empirical distribution to a theoretical distribution. The first assumption is that although our data is recorded in a discrete form - 1, 2, 4, 6, 8 hours per shift, the real activities are completed in a more continuous manner. The next assumption is that if we use a theoretical distribution, we must accept the fact that there could be an instance where a task may take longer than the largest recorded data point. This is very true for our limited number of data points, which leads to the next assumption; even though we have something less than about 70 data points for each activity, the theoretical curves indicated with this limited sample size will be representative of the future and total picture of the time distributions for these future tasks.

Once these datasets have been analyzed and summarized, a lookup table will be created and available to the conversion software. So, when the next schedule is entered into the system to be modeled, the database will look up the activity number, and a representative historical distribution will be applied from the existing dataset. It is anticipated that each new schedule will have some amount of activities that are unknown to the modeling tool. This is where the Operations Research (OR) analyst and the subject matter expert (SME) are needed. Once these new unmatched tasks are identified, the SME will attempt to review the new task details against the available library of tasks. If a similar task can be identified, it will be used. If nothing can be related, the old standard of task estimation can be used for this item in the model with a slight twist.

Recently we have been working with other simulation analysts in the spacecraft industry. The observation presented to us was that in other spacecraft modeling techniques most tasks take on a log-normal distribution, and a percentage of the mean duration can be used as the standard deviation parameter for the log-normal theoretical curve. We intend to look closely at our data to determine if this observation holds true for our datasets. If so, it lends itself to a very simple technique for getting a distribution curve for a new task in the spacecraft environment. Then, only one estimated value, the mean, is required to establish a theoretical distribution for that new unmatched task.

\subsection{Verification and Validation}

Model verification and validation is a significant concern in an automatic generation solution. Since no one individual has a personal feeling and exposure for the developed model, it is imperative to verify and validate that the automatic generated model truly represents the process. Our planned technique is to feed actual sets of data from known OPF flows and run that deterministically through the simulation. Then, using the acquired makespan values from the model, compare them statistically to the known OPF flow durations. If this technique shows a fairly constant bias, either underestimating or over estimating the real flow, changes to the gain factor could 
be made and a re-running of the validation process performed. This is a process of calibration of the simulation.

\section{CONCLUSIONS}

The literature indicates that simulation is a very effective solution to resource constrained - project scheduling problems with stochastic durations. Simulation may not identify the optimal solution, but it will be useful for estimation of a statistically sound makespan for the project. The construction business is the most commonly understood example of a resource constrained - project scheduling problem with stochastic durations. Initially, simulation modeling was not perceived to have benefits for the construction project business. With advances in PC technology that have resulted in rapid computer solutions affecting more real world practice, Construction Project Scheduling has seen benefits from the usage of simulation modeling. Automatic generation of simulation models has seen favor in the manufacturing arena. The use of autogenerated models will permit the simulation tool to find more widespread usage among the project managers. Existing scheduling tools, when combined with knowledge repositories of task timelines and distributions, contain the necessary details to produce good simulation models. Creative application and modularization of schedule details translated into simulation constructs can be used to effectively and efficiently produce simulation models for project duration analysis. Once available to the team, autogenerated simulation models from planning efforts, will be useful in accessing potential improvements in the OPF flow makespan.

\section{FURTHER RESEARCH}

The large positive correlation identified between ranking of critical activities based on the ratio of average slack to the standard deviation of activity duration to Criticality Index needs to be explored and proven. In other terms, proving that project makespan can be shortened by performing equal precedence tasks in a project network, in a ranked order by highest variability to lowest variability, would benefit the OPF Flow process and any similar resource constrained - stochastic project scheduling problem.

\section{APPENDIX - ACROYNMS}

\begin{tabular}{|c|c|}
\hline ARENA & $\begin{array}{l}\text { Arena Simulation Modeling Software - by } \\
\text { Rockwell Automation }\end{array}$ \\
\hline CAPSS & $\begin{array}{l}\text { Computer Aided Planning and Scheduling } \\
\text { System }\end{array}$ \\
\hline CHIT & Special Action Request \\
\hline CI & Criticality Index \\
\hline $\mathrm{EO}$ & Engineering Orders \\
\hline ET & External Tank \\
\hline GPSS & Ground Planning and Scheduling System \\
\hline GSE & Ground Support Equipment \\
\hline IOS & Integrated Operations System \\
\hline KBSI & Knowledge Based Systems, Inc. \\
\hline MOD & Modifications \\
\hline NASA & National Aeronautics and \\
\hline OMRSD & $\begin{array}{l}\text { Administration } \\
\text { Orbiter Maintenance \& Requirements } \\
\text { Specification Documentation }\end{array}$ \\
\hline OPF & Orbiter Processing Facility \\
\hline PRACA & Problem Recording and Corrective Action \\
\hline RCPSP & $\begin{array}{l}\text { Resource Constrained - Project Scheduling } \\
\text { Problem }\end{array}$ \\
\hline RCSPSP & $\begin{array}{l}\text { Resource Constrained - Stochastic Project } \\
\text { Scheduling Problem }\end{array}$ \\
\hline SCAN & Shuttle Connector Analysis Network \\
\hline SME & Subject Matter Expert \\
\hline $\mathrm{SRB}$ & Solid Rocket Booster \\
\hline SSP & Space Shuttle Program \\
\hline STS & Space Transportation System \\
\hline TEAMS & $\begin{array}{l}\text { Toolkit for Enabling Adaptive Modeling \& } \\
\text { Simulation }\end{array}$ \\
\hline $\mathrm{TF}$ & Total Float - Slack Time for a Task \\
\hline USA & $\begin{array}{l}\text { United Space Alliance, LLC - Joint Venture } \\
\text { by Lockheed-Martin and Boeing for Human } \\
\text { Spaceflight Operations Contracts }\end{array}$ \\
\hline VAB & Vehicle Assembly Building \\
\hline VBA & Visual Basic Application \\
\hline WAD & Work Authorization Document \\
\hline WorkSim & KBSI Modeling Software \\
\hline
\end{tabular}




\section{REFERENCES}

AbouRizk, S. M., and Wales, R. J. (1997). Combined discrete-event/continuous simulation for project planning. Journal of Construction Engineering and Management, 123(1), 11-20.

Akpan, E. O. P. (2000). Priority rules in project scheduling: a case for random activity selection. Production Planning \& Control, 11(2), 165.

Benjamin, P., Graul, M., and Erraguntla, M. (2002). Methods and tools for aerospace operations modeling and simulation: toolkit for enabling adaptive modeling and simulation (TEAMS). In the Proceedings of the 2002 Winter Simulation Conference, San Diego, California, 763-771.

Boydstun et al. (2002). Methods and tools for aerospace operations modeling and simulation: new perspectives towards modeling depot MRO. In the Proceedings of the 2002 Winter Simulation, San Diego, California, 738-746.

Brucker et al. (1999). Resource-constrained project scheduling: notation, classification, models, and methods. European Journal of Operational Research, $112(1), 3-41$.

Herroelen, W. S., \& Leus, R. (2001). On the merits and pitfalls of critical chain scheduling. Journal of Operations Management, 19(5), 559-577.

Möhring, R. H., \& Stork, F. (2000). Linear preselective policies for stochastic project scheduling. Mathematical Methods of Operations Research, 52(3), 501.

Pritsker, A. A. B., Sigal, C. E., and Hammesfahr, R. D. J. (1989). Slam II network models for decision support. Englewood Cliffs, N.J.: Prentice Hall.

Zweben et al. (1993). Scheduling and rescheduling with iterative repair. Systems, Man and Cybernetics, IEEE Transactions on, 23(6), 1588-1596.

\section{AUTHOR BIOGRAPHIES}

Our team of researchers at USA was formed using subject matter experts (SME) in the OPF Process that have extensive backgrounds in the processing of space shuttles. These individuals have decided to move into a more operations research career path. Each brings a different understanding of the problem and the historical data, which produces a well-rounded effort in the final model and simulation product.

MICHAEL G. MADDEN is a Ph.D Student in the University of Miami's - Industrial Engineering Program and a former Sr. Vehicle Engineer for Space Shuttle Endeavour and Atlantis. His subject matter expertise is from the space shuttle senior engineering group with experience working with the processing team and test team responsible for technical decisions of the shuttle process. He now works in the Florida Program Office of United Space Alliance, LLC - developing simulation technology for estimation of space shuttle processing flows. He has a MS in Technology Management from Embry-Riddle Aeronautical University, and a BS-MET from Wentworth Institute of Technology in Boston, Ma. His research interests are in scheduling and other operations research problems associated with shuttle processing and knowledge management. He is a member of the INFORMS Simulation Society, ACGIH, IEEE, and AIChE.

ROBERTA WYRICK is a Certified Lean Six Sigma Black Belt working in the Florida Program Office of United Space Alliance, LLC on the development of modeling techniques for the space shuttle process. Her subject matter expertise comes from the operations and flow management department where she was responsible for the coordination and integration of operations in the development of space shuttle schedules as the Flow Manager for Atlantis. She has a BS in Mathematics from University of Central Florida. She is a member of the INFORMS Simulation Society.

DALE E. O'NEILL is also working in the Florida Program Office of United Space Alliance, LLC on the development of modeling techniques for the space shuttle process. He was manager and member of the planning and scheduling organization for the OPF process for more than 20 years. He has a BS in Business Administration from University of Central Florida. He is a member of the INFORMS Simulation Society. 